\title{
Régulations magistrales et effets mésogénétiques: Des tensions entre dispositif et situation
}

\section{Marc Weisser}

Comment l'enseignant s'y prend-il pour réguler les échanges entre élèves et ainsi parvenir à ses fins? C'est la question que nous proposons d'approfondir dans ce texte. Nous comparons le déroulement d'une séquence d'enseignement avec le dispositif conçu a priori par le professeur. Les outils mis en oeuvre pour analyser le corpus recueilli sont empruntés à la linguistique pragmatique. Les lieux stratégiques où l'enseignant intervient pour orienter la réflexion de sa classe sont identifiés. Le rôle joué par ses institutionnalisations locales et partielles est précisé. La conclusion présente quelques réflexions sur les tensions entre dispositif pédagogique préexistant et situation vécue en classe.

\section{Exploration de l'espace-problème et présence enseignante ${ }^{1}$}

Ce texte s'intéresse aux rapports entre maître et élèves ${ }^{2}$ lors des interactions orales, c'est-à-dire à la co-construction de la situation d'enseignement que vivent ici et maintenant les protagonistes de la relation didactique. À travers l'élaboration initiale de son dispositif pédagogique, l'enseignent a organisé a priori un milieu pour apprendre (Brousseau, 1998) meublé d'objets et d'instruments, "générateur de nécessités (ressources et contraintes)" (Sensevy, 2001, p. 210) afin qu'ensuite l'élève auquel le problème aura été dévolu (Johsua, 2005) puisse s'en saisir et retrouver le savoir visé. Dans une telle approche, le contrat didactique prévoit à certains moments de constituer la classe en communauté discursive (Bernié, 2002; Swales, 1990), fédérée autour d'un projet commun de production de savoir par la mise en œuvre de débats heuristiques (Weisser, 2003). Dans ce genre de situation, les interlocuteurs se répartissent des places discursives (Cosnier \& Kerbrat-Orecchioni, 1991) qui témoignent de leur statut de dominant ou de dominé. La position haute revient à celui qui assure la progression thématique. Ici, les élèves, grâce aux choix topogénétiques (Mercier, Schubauer-Leoni \& Sensevy, 2002) de l'enseignant, grâce à son «effacement 
didactique» (Forest, 2005), sont explicitement mis en position de pouvoir explorer un «espace socio-discursif de partage des significations» (Bernié, 2002, p. 77) à l'intérieur duquel il y a nécessité de s'entendre sur la définition des objets mobilisés et sur l'élaboration finale du savoir en germe dans le dispositif magistral.

Il s'agit donc pour nous d'étudier «l'articulation des processus cognitifs, sociaux, didactiques et discursifs» (Schubauer-Leoni, 1994, p. 79) qui apparaissent dans le déroulement d'une séquence d'enseignement, et plus particulièrement à l'occasion des interactions orales. Ces temps de mise en question des productions individuelles ou de groupe nous paraissent nécessaires pour trois raisons au moins. La première est d'ordre épistémologique: "Pour ce qui est des opérations de pensée, l'enfant en train de construire ses connaissances n'est pas si différent du savant qui cherche à produire des savoirs nouveaux dans son domaine» (Leutenegger \& Saada-Robert, 2002, p. 11). Les débats sont une pratique constitutive de la recherche scientifique considérée comme une activité sociale qui vise à expliquer à travers une démarche de preuve (Orange, 2004, p. 13). La confrontation des idées des uns et des autres va amener les élèves à argumenter leur position personnelle, à étayer leurs jugements initiaux. Se construit petit à petit «un espace disponible pour ce qui, dans la classe, peut faire l'objet de doutes et de problèmes» (Lipman, 1995, p. 33). La deuxième raison relève de la psychologie. La théorie du passage de l'inter- à l'intra-personnel chez Vygotsky (1934/1985), débouchant sur l'intérêt pour les échanges entre novice et expert chez cet auteur, pour les échanges entre pairs dans le paradigme socioconstructiviste, justifie à nouveaux frais la nécessité d'instituer au sein $\mathrm{du}$ groupe classe un certain nombre de pratiques langagières qui favorisent la confrontation entre personnes et, dans le même mouvement, le progrès cognitif de chacun. La didactique peut, finalement, renforcer elle aussi notre proposition. L'activité de l'élève, même expérimentale, ne suffit pas: «L'utilisation intelligente du langage est une fonction intellectuelle qui n'est déterminée en aucune façon par une structure immédiatement perceptible à l'œil» (Vygotsky, 1934/1985, p. 121). Il faut des mots pour dire, pour décrire, des mots pour prévoir. La simple perception, même de phénomènes provoqués dans un but d'apprentissage, ne saurait garantir l'acquisition de savoirs (Weisser, 2004, pp. 112-117). De ce point de vue, le didacticien retient une approche de l'argumentation non-polémique, dans laquelle «une contradiction est souvent le moteur déclenchant une série d'opérations logico-discursives pour la surmonter, par divers remaniements et réorganisations de ce dont on parle» (Nonnon, 1996, p. 68).

Dans ce qui suit, nous focaliserons notre attention sur l'action du professeur, dans ses dimensions de régulation et d'institutionnalisation. Nous reprenons en cela deux des quatre "éléments structuraux fondamentaux» identifiés par Sensevy, Mercier et Schubauer-Leoni (2000, p. 267). De façon plus précise encore, nous chercherons à identifier les effets mésogénétiques de l'intrication entre, d'une part, la régulation des moments adidactiques de mise en commun 
des travaux individuels ou de groupe, et, d'autre part, les institutionnalisations locales, partielles, progressives auxquelles se livre simultanément l'enseignant: le maître se contente-t-il à ces moments d'archiver la pensée de la classe? A-t-il d'autres intentions?

En adoptant ce genre de démarche, l'enseignant assume un certain nombre de risques. D'un côté, celui de ne pas maîtriser par avance les découvertes, les controverses, les repentirs de ses élèves, et encore moins l'ordre dans lequel ils s'enchaînent, la durée qu'il faudra laisser à la classe pour que le problème mûrisse dans les esprits. D'un autre côté, celui de l'adoption par chacun de conduites langagières qui rendent possible la constitution d'une communauté discursive. Autrement dit, ne pas attaquer l'énonciateur, mais ses énoncés, et assumer la responsabilité de ses actes de parole (Bronckart, 1996, pp. 52-58).

Mais si l'enseignant accepte de se mettre en danger, c'est qu'il est convaincu par ailleurs que le remaniement cognitif provoqué par un apprentissage ne peut se faire par procuration (Weisser, 2008). Le professeur se contente alors de préparer le terrain en concevant des dispositifs facilitateurs, qui ouvrent à chaque apprenant un espace dans lequel il aménagera progressivement son processus de résolution de problème. Houssaye (1988) va jusqu'à dire que le maitre «fait le mort». Idée que l'on retrouve chez Brousseau dans le concept de «situation adidactique»: dans ces moments, ce n'est pas le maittre qui réagit aux sollicitations des élèves mais le milieu antagoniste (1998, p. 59), mais aussi chez Freinet: le maître n'a pas à parler, mais à avoir la maîtrise du matériel et des techniques (Invariant pédagogique $\mathrm{n}^{\circ} 20$, texte de 1942, in 1994, p. 383 et ss.).

Faire classe consiste alors à essayer de faire correspondre deux espaces. Le premier, virtuel, est celui construit par le dispositif pédagogique. Retenons pour ce terme l'acception qu'en proposent Charlier et Peeters (1999, p. 17): une articulation d'éléments hétérogènes, matériels et symboliques, moyens mis en œuvre relativement à une fin, consciente au moins dans l'esprit de celui qui conçoit et pilote le dispositif (Meunier, 1999, p. 84). Reste alors à le mettre en œuvre. Le deuxième espace, nous choisissons de le nommer situation, en référence à Sartre, pour qui l'homme n'est libre qu'en situation, engagé dans un monde résistant meublé d'objets dont le coefficient d'adversité / de facilitation n'est qu'une conséquence de nos projets à leur égard (1943, pp. 577 et 538; voir Weisser, 2010). Effectivement, l'élève, «en situation», va jouer contre un milieu antagoniste (Brousseau, 1998) et finalement gagner contre lui grâce à la (re-) découverte d'un savoir. La situation est alors définie à la fois par des caractéristiques externes objectives s'originant principalement dans le dispositif magistral auquel elle réfere, mais aussi par la manière dont chaque sujet apprenant l'interprète (Schubauer-Leoni, 1996, pp. 165-168).

D'où notre questionnement pour le présent article: quel est ce mode de présence / absence de l'enseignant dans les moments adidactiques? Comment s'y prend-il pour être ce «mort efficace» (Weisser, 2008) qui laisse ses élèves libres d'explorer l'espace de la situation, mais qui en même temps a la ferme intention 
de les accompagner vers ce que prévoit son dispositif? Certains parlent parfois de "pratiques ostensoires déguisées» (Matheron \& Salin, 2002), quand le maître sort clandestinement de sa réserve en mettant en avant un objet du milieu didactique; d'autres invoquent la "postulation mimétique» (Sensevy, 2001, p. 223), quand il s'imagine, à tort, ou prématurément, que l'élève a compris ce qui était requis. En tout état de cause, il nous semble pertinent d'étudier en détail quel est le degré d'intervention de l'enseignant dans sa gestion des moments d'argumentation heuristique, sur quels plans il intervient (mésogenèse, chronogenèse, topogenèse). Et quels sont en conséquence les effets s'agissant des apprentissages opérés par les élèves.

L'entrée par la didactique comparée (Mercier et al., 2002) à travers son triplet conceptuel nous semble en effet prometteuse. Nous définirons la mésogenèse comme une suite organisée d'objets de savoir et d'états de ces objets (Sensevy, 2001, p. 209), comme le processus qui associe successions, coexistences, substitutions d'objets physiques ou symboliques au fil de la séquence (Schubauer-Leoni, 2003). La chronogenèse figure cette succession d'états d'un système d'objets culturels enseignés, marquant le temps didactique (Mercier et al., 2002, p. 10). La topogenèse quant à elle souligne l'évolution des positions adoptées par le professeur et ses élèves durant la séquence didactique, l'objet de l'enseignement étant de faire changer les rapports au savoir de chacun (Johsua, 1996, p. 155).

C'est donc toute la communication didactique, en tant que recherche d'une compatibilité, voire d'une conformité "entre le nouveau rapport de connaissance [établi par l'apprenant] et le rapport de savoir visé par l'institution" (SchubauerLeoni, 2003), qui doit faire l'objet de l'attention du chercheur. Les théories convoquées pour cet exercice, qu'elles relèvent de la linguistique, de la pragmatique, de la logique ou d'autres champs proches (voir Vion, 1999, pp. 43-47), s'appliquent toutes à mettre en évidence les mécanismes de médiation entre pairs, entre néophyte et expert, entre apprenant et savoir. Il est de surcroît significatif de relever leur convergence épistémologique avec les travaux des didacticiens, et avec ceux du champ de la psychologie s'intéressant aux processus interprétatifs, menés dans une perspective néo-piagétienne (Schubauer-Leoni, 2003). Tous en effet mettent l'accent sur la négociation progressive du sens de ce qui s'échange, sur l'influence du discours d'autrui, sur les ressorts de l'argumentation. Sensevy et Quilio (2002, p. 47) vont jusqu'à jeter les bases d'une «pragmatique didactique», fondée sur les effets contextuels et les calculs d'inférences auxquels se livrent les protagonistes d'une séquence d'apprentissage.

Dans ce qui suit, nous présentons tout d'abord nos outils méthodologiques, par conséquent empruntés à la linguistique pragmatique. Nous rendons compte ensuite de notre propre dispositif, expérimental. L'analyse du corpus ainsi construit nous permettra finalement de revenir sur notre question initiale des tensions entre dispositif pédagogique et situation d'enseignement. 


\section{Rendre compte de ce qui se dit en classe}

Nos hypothèses du chapitre précédent nous amènent à nous centrer sur l'enseignant. On ne distinguera pas les sujets apprenants entre eux, notre projet n'étant pas ici, par exemple, de relier des types de participation, des fréquences de tours de parole, avec les acquisitions réalisées en fin de séquence (pour une focalisation sur les élèves, voir entre autres Weisser, 2006, 2009). Nous retenons l'entrée par la linguistique pour rendre compte de la polygestion des interactions orales, depuis l'expression des subjectivités et leur interprétation par les interlocuteurs, jusqu'à la négociation du sens qui en résulte. Nous tâcherons de mettre en évidence les systèmes de normes régulatrices des interactions, telles qu'elles préexistent, telles qu'elles sont redéfinies chemin faisant (Schubauer-Leoni, 1994, p. 80).

Dans un premier temps, il s'agit de repérer les articulations au sein de la séquence d'enseignement observée. Avec Moeschler et Reboul (1994, p. 479), nous retenons quatre unités d'analyse incluses les unes dans les autres. La plus large, baptisée incursion, figure l'interaction dans son ensemble. Par conséquent, chaque séance observée correspond à une nouvelle incursion. Sous-ordonnée à la précédente, la transaction réunit un ensemble d'échanges présentant une unité thématique. Le principe de récursivité (Moeschler \& Reboul, 1994, p. 482) autorise l'enchâssement des transactions, en d'autres termes, la prise en compte de sous-thématisations. À un grain plus fin, la première unité de rang monologal, l'intervention (ou tour de parole), peut être à son tour subdivisée en actes de langage. L'application de cette grille d'analyse débouche sur la mise au jour du déroulement de la séquence: quels objets ont été successivement thématisés? Sous lesquels de leurs aspects? La classe, étayée par son enseignant, a-t-elle suivi un chemin rectiligne? A-t-elle emprunté des itinéraires ramifiés ? Ou, dit autrement, comment la situation collégialement construite a-t-elle incarné le dispositif magistral préexistant?

A partir de là, nous allons décrire les interventions du professeur. La logique interlocutoire (Trognon, 1994, pp. 20-24) vient à notre aide en distinguant dans chaque acte de langage son contenu propositionnel et sa force illocutoire. Le contenu propositionnel reflète l'objet dont il est question (thématisation) et ce qui en est dit (sous-thématisation); la force illocutoire traduit ce qu'on en fait. On a ainsi caractérisé les assertifs (le locuteur engage sa responsabilité sur un état du monde), les commissifs (il s'engage sur un comportement à venir), les directifs (il cherche à faire faire ou dire quelque chose à ses interlocuteurs), les expressifs (il exprime un état psychologique personnel) et les déclaratifs (il modifie un état du monde par ce qu'il dit) (Trognon, 1999). Comme on le voit, un même contenu propositionnel peut être mis en scène de bien des façons, avec en tout cas des intentions différentes: un commissif tendra, le cas échéant, à initier une démarche commune qu'on se promet de mener jusqu'à son terme, tandis qu'un déclaratif refermera au contraire toute velléité de recherche. 
Il nous faut ajouter pour être complet qu'un acte de langage vise de plus à être réussi et satisfait (Trognon, 1994, p. 21): il est réussi si sa force illocutoire est reconnue comme telle (un enseignant donne une directive à un élève, celui-ci le comprend); il est satisfait si de plus l'interlocuteur y répond de façon conforme (l'élève a compris la directive et il s'y plie). Il ne suffit pas en effet de dire quelque chose pour être entendu, et encore moins pour que le message transmis soit suivi d'effet (voir Weisser, 2007). Cette seconde strate d'analyse a pour but de caractériser les effets mésogénétiques des régulations magistrales: quelle est la part de responsabilité de l'enseignant dans la gestion des écarts qu'il constate entre la situation ici et maintenant et le dispositif qu'il a patiemment conçu? Quel degré de conscience a-t-il de ses actes de langage, sachant qu'il est contraint d'agir (et de réagir) en temps réel, soumis à quantité d'informations dont la caractéristique principale n'est pas la cohérence?

Mais avant de passer à cet examen minutieux de ce qui se dit en classe, il nous reste à brosser à grands traits notre dispositif expérimental.

\section{Un problème un peu particulier}

Notre groupe de recherche a fourni un même problème à six professeurs des écoles de Cours Moyen $2^{\mathrm{e}}$ année (enfants de 10 ans). Il s'agit d'une photographie ${ }^{3}$ accompagnée de l'énoncé suivant: «Cette photo a été prise dans un parc d'attractions en Angleterre. On y aperçoit une partie de la jambe d'un géant. Quelle est la taille de ce géant?» (Document 1).

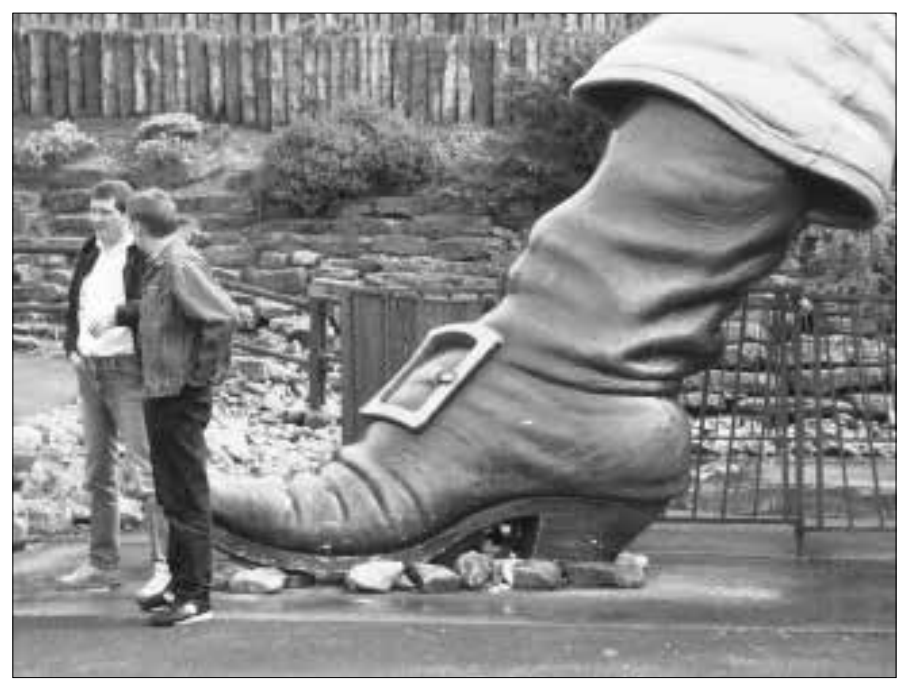

Document 1: photographie remise aux enseignants 
Il ne saurait là être question d'ingénierie didactique, les informations transmises étant des plus laconiques. Nous avons donc affaire à des "classes ordinaires», au sens où les séances n'ont fait l'objet d'aucun travail préalable approfondi entre les chercheurs et l'enseignant (Margolinas, 2005, p. 350). Ce support présente un certain nombre de particularités mathématiques:

Aucun nombre n'est fourni dans l'énoncé, résoudre le problème nécessite de faire des hypothèses (taille d'un homme, taille du pied d'un homme, proportions du géant, etc.), le problème est ouvert (la réponse dépend des hypothèses et des mesures éventuellement prises sur la photo), personne ne connaît la «bonne» réponse, ni les élèves, ni le maître, ni les chercheurs, la réponse ne peut être fournie qu'au moyen d'une estimation, par exemple sous la forme d'une fourchette (Rauscher, Adjiage \& Beliaeva, 2009).

Nous faisons l'hypothèse que ce genre de problèmes non-standards, en ce qu'ils déstabilisent quelque peu les habitudes des enseignants, accentuent leurs options pédagogiques, rendent les observations plus parlantes.

Trois stratégies de résolution ont été identifiées (Rauscher, Adjiage \& Beliaeva, 2009). La première s'appuie sur le fait que l'homme arrive à peu près à la moitié du mollet du géant. On va donc chercher le rapport entre le mi-mollet et le corps entier chez les hommes, faire l'hypothèse que le géant obéit aux mêmes proportions, et multiplier la taille (hypothétique) de l'homme par ce rapport, pour trouver la taille du géant. La deuxième solution compare d'abord les longueurs des semelles de l'homme et du géant, mesurées sur la photo. Si la botte du géant est $n$ fois plus grande que la chaussure de l'homme, c'est que le géant lui-même est $\mathrm{n}$ fois plus grand que l'homme (hypothèse de la constance du rapport personne / semelle). Enfin, la troisième stratégie commence par calculer la taille du géant sur la photo, par exemple en posant les rapports personne I semelle et en calculant la $4^{\mathrm{e}}$ proportionnelle. Ensuite, on trouvera la taille réelle du géant de la même façon, en posant les rapports personne sur la photo / personne réelle, avec là encore une taille hypothétique (1,80 m par exemple) pour l'homme représenté. Ce type de problèmes de proportionnalité, les dilatations (agrandissements ou réductions) présente des difficultés tout à fait spécifiques et engendre un faible taux de réussite (Adjiage, 2005, p. 106).

Chacun des six enseignants observés a, à partir de cela, dû concevoir son dispositif pédagogique, en termes de nombre de séances, de durée, d'organisation de la succession des phases de travail, de confection ou sélection des outils pour leurs élèves, etc. ${ }^{4}$

La classe retenue pour la présente étude accueille vingt-deux élèves, en milieu populaire urbain. Nous y avons recueilli trois séries de données. Deux semaines avant la mise en situation, il a été demandé à l'enseignant de résoudre lui-même le problème et de nous retourner son écrit accompagné de sa fiche de préparation; les élèves ont été soumis à un pré-test portant sur les différents aspects de la proportionnalité. La séquence en elle-même a été filmée puis retranscrite; les écrits de travail des élèves ont été photocopiés, ainsi que leur résolution indivi- 
duelle finale du problème du géant. Après coup, le maître a été questionné sur le bilan qu'il tirait de son action et les élèves ont été soumis aux mêmes exercices qu'initialement, à titre de post-test. Ce dispositif expérimental en trois temps est analogue à ceux que l'on trouvera par exemple chez Sensevy (2001, p. 213) ou chez Schubauer-Leoni et Chiesa Millar (2002, pp. 123-124). Son atout principal est, justement, de permettre l'analyse des (éventuels) écarts entre dispositif pédagogique et situation d'enseignement, entre intentions magistrales préalables et gestion de la classe en temps réel. Ne nous plaçant pas dans une optique d'évaluation des acquis mais bien plus d'analyse des tractations entre maître et élèves, nous ne ferons pas appel ici aux résultats des pré- et post-tests.

\section{Au fil des thématisations}

Comme indiqué dans nos options méthodologiques, nous allons pour commencer tenter de retrouver les articulations de la séquence d'enseignement à travers l'identification des thématisations qui ont successivement retenu l'attention de la classe. L'enseignant observé a choisi d'organiser deux séances pour amener ses élèves à résoudre le problème du géant, et c'est effectivement ce qu'il a réalisé. Nous allons par conséquent détailler deux incursions distinctes d'une durée d'environ soixante minutes chacune. Nos transactions seront subordonnées à une consigne magistrale, ce qui fixe à chaque fois le type d'interaction (individuelle avec l'énoncé du problème, en petits groupes, en classe complète) et définit le thème (chercher, mesurer, calculer, communiquer, prouver, transférer). Les sous-transactions seront quant à elles identifiées autour du traitement d'un objet particulier du milieu didactique, celui qui, à cet instant-là, est jugé pertinent par la classe, traduisant la sensibilité momentanée des élèves à l'une des caractéristiques de la situation-problème.

La première incursion peut se subdiviser en cinq transactions:

- Transaction 1.1.: mise en route de l'activité, recherche individuelle sur le problème: $7 \mathrm{M}^{6}$ «Vous écrivez ce que vous trouvez, hein, s'il y a des idées qui vous viennent» (0 à 8 min 40).

- Transaction 1.2.: mise en commun des travaux individuels (8 min 40 à 23 $\min )$.

- Transaction 1.3.: travail de groupe: $132 \mathrm{M}$ «Vous allez discuter et vous allez mettre tout ce qu'on sait quand même grâce à l'image, même si on n'a pas l'énoncé comme d'habitude" (23 min à $30 \mathrm{~min}$ ).

- Transaction 1.4.: mise en commun des travaux de groupes (30 min à 41 $\min )$.

- Transaction 1.5.: «Exercices pour se préparer à résoudre le problème du géant»: trois exercices composés par l'enseignant; à chaque fois, réflexion personnelle puis correction en commun (47 min à $57 \mathrm{~min}$ ). 
La seconde incursion ne comporte quant à elle que quatre transactions:

- Transaction 2.1.: rappel des acquis de la séance précédente (0 à 5 min 30).

- Transaction 2.1.: travail de groupe: proposer une solution au problème (5 $\min 30$ à $30 \mathrm{~min}$ ).

- Transaction 2.3.: mise en commun des travaux de groupes (30 min à 52 $\min )$.

- Transaction 2.4.: travail individuel de résolution finale (52 min à $60 \mathrm{~min}$ ).

La démarche pédagogique mise en œuvre alterne systématiquement des moments de recherche, individuelle ou en groupe de trois ou quatre élèves, et des moments de mise en commun. L'enseignant fait construire le savoir en s'appuyant à chaque fois sur des interactions entre apprenants, à propos de questions sur lesquelles ils ont préalablement pris position par écrit. Chacune de ces phases dure entre dix et vingt minutes en général, ce qui évite la lassitude et fait régulièrement rebondir l'intrigue par l'apport de perturbations extérieures.

Nous allons nous intéresser de plus près à ces épisodes polygérés parce que, focalisé sur l'enseignant, nous y trouvons les traces de son activité de gestion de l'avancée du temps didactique, et parce que, conformément à ce que nous avons annoncé plus haut, c'est dans ces temps de débat heuristique que l'on peut observer l'articulation des processus cognitifs et sociaux par le truchement du discours.

La transaction 1.2. est la première de ce type. Le maître l'ouvre par l'énoncé suivant: $10 \mathrm{M}$ «Comment vous avez abordé ce problème ou quels sont les soucis que vous rencontrez?» Un élève saisit la balle au bond: $11 \mathrm{E}$ «Euh on ne peut pas résoudre ce problème...» C'est donc l'impossibilité de l'action qui est thématisée initialement. La raison en est invoquée immédiatement: 13E «Pour savoir la taille du géant il faut d'abord voir l'image en entier, voir comment il est d'abord». Mais un autre élève prend l'initiative d'introduire un nouveau thème: $21 \mathrm{E}$ «Moi je pense que pour commencer le problème il faut déjà commencer par savoir combien de fois le géant est plus grand, et on regarde si c'est normal». La discussion glisse alors sur la taille de l'homme qui se tient à ses côtés: $25 \mathrm{E}$ «La taille de l'homme on peut l'approximer euh...", 28M "ça vous semble vrai ce qu'il dit?», 29E «Oui parce que tous les gens mesurent à peu près la même taille». La classe s'accorde sur une fourchette qui va de $1,75 \mathrm{~m}$ à $1,80 \mathrm{~m}$. L'enseignant donne ensuite explicitement la parole à un autre élève: $54 \mathrm{M}$ "'’aurais aimé que tu nous lises ce que tu as écrit s'il te plaît», $55 \mathrm{E}$ « Euh déjà je cherche à peu près la taille du géant». A partir de là, tout le groupe va recenser les raisons de cette incertitude: on ne voit pas le géant de face mais de biais (79E), on ne dispose pas de données numériques (83E), on ne voit qu'une partie du géant (91E), on n'a qu'une taille approximative pour l'homme de la photo (92M). Les deux sous-thématisations suivantes sont stoppées par l'enseignant: l'un des élèves présente sa démarche de résolution, le maître lui répond de garder son idée pour une phase ultérieure de la séquence; un autre fait allusion au manège également visible sur 
l'image, il lui est signifié qu'ici non plus, aucun renseignement supplémentaire n'est disponible. La transaction s'achève sur le thème des problèmes analogues. C'est là encore un élève qui l'introduit, spontanément: 105E «Faudrait peut-être une échelle pour trouver la taille du géant». Cette fois-ci, l'enseignant reprend le propos à son compte: $106 \mathrm{M}$ "Toi tu aurais aimé... On n'a pas d'échelle. Ça te fait penser à quel... à quel problème ça?». La classe liste alors des types rencontrés plus tôt dans l'année: problèmes d'échelle, de cartes, de proportionnalité, et en donne à chaque fois des exemples. C'est finalement l'enseignant qui clôt l'échange en lançant le travail de groupe (transaction 1.3., voir ci-dessus). A ce moment-là de la séquence, le tableau noir, renseigné au fur et à mesure par le professeur, présente l'aspect suivant:

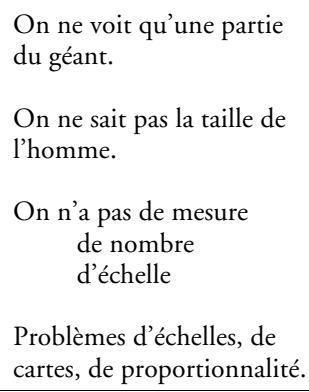

Après le travail de groupe qui a pour but d'identifier toutes les informations que l'on peut retirer de l'image, le maître anime un deuxième débat: $141 \mathrm{M}$ «Alors qu'est-ce que vous savez de plus? Quelque chose qu'on savait pas au départ mais qu'on a pu découvrir?». Réponse immédiate: $142 \mathrm{E}$ «1 mètre 80 égale huit centimètres». Ces deux valeurs sont ensuite discutées, tant au plan de leur exactitude qu'en ce qu'elles représentent. L'enseignant inscrit finalement au tableau, volet central: «Les deux hommes mesurent 1,80 $\mathrm{m}$ (?) en réalité, $8 \mathrm{~cm}$ sur la photo» et clôt l'échange: $163 \mathrm{M}$ «Bon, ça, c'est bien, on sait déjà quelque chose». Un élève s'autorise alors (164E) à donner sa longueur du pied du géant: $9 \mathrm{~cm}$. La classe négocie successivement le résultat (arrondi ou pas?) et l'orientation de la mesure (horizontale ou de biais? la semelle ou la tige de la botte?). La décision prise collégialement est ensuite reportée au tableau. Pour finir, c'est la longueur des chaussures des hommes de la photo qui est thématisée, là encore par un élève, qui répond spontanément à l'invite du maître: $191 \mathrm{M}$ «Est-ce que vous avez mesuré d'autres choses?». La précision de la mesure est contestée, puis le rapport taille / pointure fait l'objet d'un débat. On s'entend finalement pour dire qu'à l'échelle de la photographie, les variations de pointure sont peu perceptibles. La taille de la semelle sur l'image est inscrite au tableau, qui présente la configuration suivant en fin de séance: 


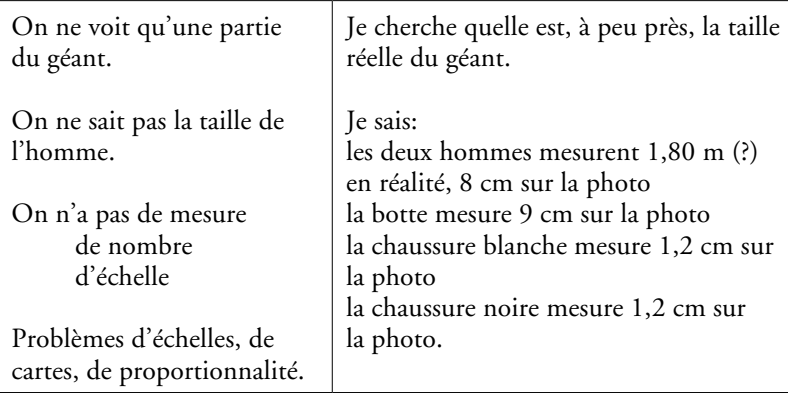

Je cherche quelle est, à peu près, la taille réelle du géant.

Je sais:

les deux hommes mesurent $1,80 \mathrm{~m}$ (?)

en réalité, $8 \mathrm{~cm}$ sur la photo

la botte mesure $9 \mathrm{~cm}$ sur la photo

la chaussure blanche mesure $1,2 \mathrm{~cm}$ sur

la photo

la chaussure noire mesure $1,2 \mathrm{~cm}$ sur

la photo.

Pour le début de la seconde séance, le tableau noir est remis dans l'état ci-dessus, ce qui autorise une rapide phase de révision. A partir des données numériques ainsi remises en mémoire, les élèves sont conviés à résoudre le problème, au sein des mêmes groupes que précédemment. Nous nous intéresserons une fois encore au moment de mise en commun de ces travaux. Cette transaction, 2.3., est ouverte par l'enseignant. Il introduit d'emblée le thème des variations du résultat: $61 \mathrm{M}$ «Est-ce qu'on peut l'admettre, ça, que les résultats sont un peu différents?». La classe acquiesce collectivement, certains explicitent leur position: E66 «Parce que on fait peut-être pas les mêmes calculs», E68 «On n'a pas les mêmes idées», E70 "On n'a pas les mêmes mesures». L'enseignant introduit alors un outil pour la résolution du problème: 73M: «Alors moi, j'ai vu beaucoup de gens, beaucoup, beaucoup, faire ceci [dessine une grande croix au tableau]. Et me dire, ah, mais c'est une règle de trois, ça va marcher. Mais après, on ne savait pas toujours ce qu'on voulait mettre dans le tableau, c'était pas facile. Alors... qu'est-ce qu'on a pu écrire dans le tableau?». Le débat porte alors successivement sur les quatre emplacements du produit en croix. La taille de la chaussure de l'homme apparaît d'abord (74E); l'enseignant demande s'il s'agit de la taille réelle ou de la taille sur la photo; c'est la seconde proposition qui est reprise (78E), en cm (80E). Un élève vient ensuite de son propre chef placer la taille de la botte dans le tableau de proportionnalité: $9 \mathrm{~cm}$. $85 \mathrm{M}$ «Qu'est-ce qui peut encore nous servir?»; $86 \mathrm{E}$ «La taille de l'homme». Le maitre note ainsi le libellé de la troisième case. 87M "Qu'est-ce que j'écris?»; 90E «1,80 m». Cette mesure est ensuite convertie en $\mathrm{cm}$ et notée au tableau. L'enseignant oriente alors l'interaction vers la recherche du coefficient de dilatation. Il se réfere pour commencer à l'un des exercices qu'il a composés pour cela (cf. transaction 1.5.), dans lequel il s'agissait de comparer les tailles respectives d'un personnage et d'un arbre $(x 4 ; x 3 ; x 3,5)$ : $99 \mathrm{M}$ «X, tout à l'heure, elle m'a dit la botte est plus grande que quoi?»; 100E «Que la chaussure»; $105 \mathrm{M}$ «Qu'est-ce qu'on peut chercher?»; 106E «Combien de fois la chaussure de l'homme est dans la botte du géant». Diverses procédures sont mises en place pour trouver le rapport qui lie 1,2 et 9 (par multiplications approchées, par division). On retient un coefficient de 7,5 (150E). Ce qui donne finalement au tableau noir, volet droit: 


\begin{tabular}{c|c} 
chaussure photo & botte photo \\
1,2 & 9 \\
\hline taille de l'homme & \\
180 &
\end{tabular}

Les propriétés du tableau de proportionnalité sont activées et 160E complète oralement: «On n'a qu'à multiplier 7,5 par la taille de l'homme et on va rejoindre le géant». L'enseignant insiste sur ce rapport multiplicatif, fait le lien avec un autre des exercices de la fin de la séance précédente (autre cas de dessin incomplet). Il fait ensuite le tour des groupes qui n'ont pas pris la parole pour leur demander leur démarche: $171 \mathrm{M}$ «Alors quels sont ceux qui ont réussi à résoudre?». Une deuxième procédure est validée, fondée sur le rapport à mi-mollet entre l'homme et le géant. Le coefficient de dilatation calculé est cette fois-ci de 8 . Un troisième exemple, proposé par un autre groupe, fait ensuite l'objet d'un traitement écrit sur le volet gauche du tableau noir. Il met en relation les mesures de la chaussure et de la botte sur la photo, et la taille de l'homme, toujours sur la photo:

\begin{tabular}{c|c}
1,2 & 9 \\
\hline 180 &
\end{tabular}

L'enseignant conclut par 237M «Alors, si on trouve combien de fois l'homme est plus grand que sa chaussure, peut-être qu'on arrivera après à trouver euh... Et le problème, c'est que vous êtes sur la photo, là. Vous saurez la taille du géant...?»; $238 \mathrm{E}$ «...sur la photo»; 239M «Sur la photo. C’est un peu embêtant...» Cet échange marque la fin de la transaction 2.3. Pour passer à l'évaluation individuelle finale (transaction 2.4.), le professeur ferme le volet droit du tableau, où figure le premier produit en croix, et ajoute la date et le titre sur le volet gauche. Le tableau s'organise alors comme suit:

\begin{tabular}{|c|l|l|l|}
\hline Lundi 17 mai 2010 & $\begin{array}{l}\text { Je cherche quelle est, à peu près, la taille } \\
\text { réelle du géant. }\end{array}$ & \multicolumn{2}{|c|}{ x 7,5} \\
Mathématiques & $\begin{array}{l}\text { Je sais: } \\
\text { les deux hommes mesurent } 1,80 \mathrm{~m}(?) \\
\text { en réalité, } 8 \mathrm{~cm} \text { sur la photo } \\
\text { la botte mesure } 9 \mathrm{~cm} \text { sur la photo } \\
\text { la chaussure blanche mesure } 1,2 \mathrm{~cm} \text { sur } \\
\text { la photo } \\
\text { la chaussure noire mesure } 1,2 \mathrm{~cm} \text { sur } \\
\text { la photo. }\end{array}$ & $\begin{array}{l}\text { botte photo } \\
\text { photo }\end{array}$ & 9 \\
\hline \begin{tabular}{l|l|l|} 
taille de \\
l'homme \\
180
\end{tabular} & \\
\hline 8 & 9 & \\
\hline
\end{tabular}

(volet rabattu au début de la transaction 2.4.) 
Cette description, un peu laborieuse, de la situation telle qu'elle s'est déroulée, nous a permis de repérer les différentes péripéties des interactions entre le maître et sa classe. Il nous faut maintenant examiner de plus près comment l'enseignant s'y prend pour exercer une influence sur ses élèves-chercheurs et comment, par conséquent, il parvient à ses fins.

\section{Interventions magistrales}

L'objectif annoncé à l'instant ne peut aboutir que si nous disposons de données auxquelles comparer le déroulement de la situation. Celles-ci existent en particulier dans la fiche de préparation du professeur. A la lecture de ce document, il apparaît que deux séances d'environ une heure étaient effectivement annoncées. La première prévoyait de commencer par un temps individuel de réflexion sur le problème du géant. Ce qui a bien eu lieu (transaction 1.1., voir ci-dessus). Une mise en commun devait s'ensuivre (transaction 1.2.), puis un nouveau travail de recherche, en groupe cette fois-ci, avec un temps de confrontation (transactions 1.3. et 1.4.). La séance devait s'achever par des exercices faits individuellement et corrigés dans la foulée (transaction 1.5.). Comme on le voit, l'ensemble des phases conçues a priori a été réalisé, dans les modalités et l'ordre prédéfinis. Il en va strictement de même pour la seconde partie de la séquence.

Le dispositif semble bien avoir été incarné comme prévu par la situation vécue en classe. Ses qualités intrinsèques initiales y suffisent-elles? L'enseignant a-t-il de plus dû intervenir en situation pour canaliser la réflexion des élèves? Un premier phénomène intéressant apparaît si l'on s'intéresse aux interventions, premières unités de rang monologal. C'est en effet une intervention du professeur qui, systématiquement, ouvre et clôt chacune des transactions:

- Transaction 1.1.:

$3 \mathrm{M}$ «Vous écrivez votre prénom sur la feuille en haut à gauche tout de suite sur la feuille blanche [...] Vous écrivez ce que vous trouvez hein»;

$10 \mathrm{M}$ «Bon [frappe dans ses mains] vous posez le stylo».

- Transaction 1.2.:

$10 \mathrm{M}$ «Maintenant j'aimerais savoir... comment vous avez abordé ce problème, ou quels sont les soucis que vous rencontrez»;

$132 \mathrm{M}$ «Alors maintenant on va se mettre par groupe. On ne voit qu'une partie du géant, on ne sait pas la taille de l'homme, on n'a pas de mesures, on n'a pas de nombres, mais on va quand même essayer de s'en sortir. Vous allez discuter et vous allez mettre tout ce qu'on sait quand même grâce à l'image, même si on n'a pas l'énoncé comme d'habitude.»

Et ainsi de suite pour tout le corpus. On notera au passage qu'une seule intervention a en général pour but de clore un épisode et dans la foulée d'ouvrir le suivant. 
Par la suite, et si l'on se rappelle du principe de récursivité mentionné plus haut, il nous a été possible à un grain plus fin de caractériser les sous-thématisations, donc des transactions enchâssées dans les précédentes. A ces moments-là, les opérations d'ouverture sont prises en charge par les élèves, qui s'emparent ainsi des positions topogénétiques hautes et assurent le choix et la progression thématiques:

- Sous-transaction 1.2.1.:

$11 \mathrm{E}$ «Euh on ne peut pas résoudre ce problème...»

- Sous-transaction 1.2.2.:

$21 \mathrm{E}$ «Il faut déjà commencer par savoir combien de fois le géant est plus grand.»

- Sous-transaction 1.2.3.:

$25 \mathrm{E}$ «La taille de l'homme on peut l'approximer euh...»

Etc.

- Sous-transaction 1.4.1.:

E142 «1 mètre 80 égale huit centimètres.»

- Sous-transaction 1.4.2.:

E164 «La longueur du pied mesure 9 centimètres.»

- Sous-transaction 1.4.3.:

E192 «La taille des hommes.»

- Sous-transaction 1.4.4.:

E194 «La taille des chaussures, euh... les hommes.»

Qu'est-ce à dire? Il apparaît que ce qui importe à l'enseignant, c'est, d'une part, que les phases de la séquence qu'il a prévues dans sa préparation a priori se succèdent dans l'ordre voulu (les différentes transactions). C'est en effet dans cette alternance que s'incarnent ses choix didactiques (succession des moments de recherche, de communication, de validation, d'institutionnalisation) et pédagogiques (variation des modes de regroupement, mises en relation matériel I consigne). Mais c'est aussi, d'autre part, que les élèves constitués en communauté discursive interagissent dans les moments de débat heuristique à partir de leurs propres positions et non de celles du maitre, ou pire, de celles qu'ils lui attribuent. Ces communautés se définissent en effet par la poursuite de buts communs explicites, dont la production de connaissances, grâce à des interactions entre pairs (Swales, 1990, pp. 24-27) qui témoignent de leur investissement topogénétique. Les élèves ont de ce second point de vue à répondre, à travers les sous-transactions, aux sollicitations du dispositif, traduit en milieu pour apprendre, meublé d'objets (la photographie, les exercices, mais aussi les outils de mesure, les connaissances préacquises, ...). En d'autres termes, l'enseignant, s'il ne sait pas ce que va dire tel élève ou tel groupe quand il lui donne la parole, sait par contre ce qui va sans doute se dire à un moment ou à un autre de la transaction. Il ne s'ingénie donc plus à ouvrir les sous-thématisations, il veille juste à ce que chacune de celles que le dispositif suggère ou nécessite fasse effectivement, quelque part pendant la transaction, l'objet d'un débat entre appre- 
nants. Il n'est pas question, à ce niveau d'analyse, d'ordre de succession, mais juste d'occurrences. Que l'on parle d'abord de la longueur de la botte ou de celle de la chaussure, de la taille réelle d'un homme ou de la taille réduite de celui de la photo, n'a finalement aucune importance; l'essentiel, c'est qu'on ait évoqué l'un et l'autre aspect durant la transaction.

Ceci étant établi, force nous est cependant de reconnaître que les sous-transactions, ouvertes par les élèves, sont elles aussi refermées par le maître, qui semble ainsi déroger au principe que nous venons d'énoncer:

- Sous-transaction 1.4.1.:

$163 \mathrm{M}$ «Bon, ça, c'est bien, on sait déjà quelque chose.»

- Sous-transaction 1.4.2.:

$191 \mathrm{M}$ «On retiendra que c'est dans ce sens [que nous mesurerons la botte]. Voilà. Est-ce que vous avez mesuré d'autres choses?»

- Sous-transaction 1.4.3.:

$193 \mathrm{M}$ «Ça, on l'a écrit, hein.»

- Sous-transaction 1.4.4.:

$231 \mathrm{M}$ «Ok? Bon, tout ça, vous allez bien le garder pour cet après-midi. [...] Je vais vous donner des exercices qui vont peut-être vous aider à débloquer la situation.»

Cet état de fait tient selon nous à une autre raison que celle évoquée précédemment (respect des choix didactiques et pédagogiques), et que nous qualifierons d'épistémologique. Il s'agit de la question de la significativité d'un résultat. Et elle se pose déjà au scientifique, comme le rappelle Galison (2002): quand décider qu'une expérience s'achève, qu'un résultat probant, définitif, a été obtenu? Ce sont, à ce niveau, des discussions au sein de la communauté des chercheurs qui le permettent. Le même phénomène se produit à l'échelon scolaire dans le débat de preuve qui suit les phases de recherche. Et le risque est grand de voir l'interaction s'enliser, tourner en rond. C'est pour cette raison que l'enseignant, qui maîtrise déjà le savoir à (ré-)inventer, cède à la nécessité d'intervenir quand il estime qu'un thème a été suffisamment travaillé. Il va pour cela institutionnaliser un acquis local et partiel, nous y reviendrons. Le danger est bien sûr de clore une sous-transaction trop tôt en attribuant aux élèves des acquis qu'ils n'ont pas encore totalement construits et maîtrisés.

Après avoir étudié la façon dont le professeur gère l'avancée du temps didactique, voyons comment il organise ses relations aux individus. Prélevons quelques exemples significatifs dans la transaction 2.3., qui a pour objectif de débattre des différentes solutions au problème du géant trouvées en petits groupes:

$65 \mathrm{M}$ «Pourquoi est-ce qu'on ne pouvait pas avoir un résultat exact aujourd'hui?» $66 \mathrm{E}$ «Parce qu'on fait peut-être pas les mêmes calculs.»

$67 \mathrm{M}$ «Pourquoi est-ce qu'on ne fait pas forcément les mêmes calculs?» $68 \mathrm{E}$ «On n'a pas les mêmes idées.»

$69 \mathrm{M}$ «Oui, ça c'est vrai, on n'a pas les mêmes idées.» 
$70 \mathrm{E}$ «On n'a pas les mêmes mesures.»

$71 \mathrm{M}$ «On n'a pas les mêmes mesures, oui.»

L'enseignant semble étrangement pris de psittacisme. Quel intérêt trouve-t-il à ainsi répéter les interventions des élèves? La réponse que nous proposons concerne les opérations topogénétiques. Le maitre reprend les contenus propositionnels thématisés par les apprenants. Mais, à travers ces mêmes mots, leur applique-t-il encore la même force illocutoire (voir chapitre "Rendre compte de ce qui se dit en classe» ci-dessus)? 66E est un assertif, l'élève qui en endosse la responsabilité s'engage sur un état du monde, sur le travail de groupe qui précède. Notons au passage que la modalisation en "peut-être» et la prise en charge énonciative par "on» (nous) constituent autant d'ouvertures à autrui d'un espace de négociation. 67M reprend ce «on», il s'engage lui-même aux côtés de ce groupe, il le soutient, officialise sa procédure de résolution. Cette question cache en fait un déclaratif, l'enseignant modifie un état du monde juste par le fait de (re-)prononcer ces mots: l'élève et ses camarades sont reconnus comme dignes d'intérêt, leur travail mérite d'être discuté par les autres. 68E ne s'y trompe pas, l'acte de langage est réussi et satisfait, puisque ce nouvel énoncé constitue une réponse pertinente. $69 \mathrm{M}$ reprend le même procédé, cette reformulation littérale est un nouveau déclaratif qui vise à favoriser cette fois la rotation des positions discursives autres: chacun a droit à la parole et a le droit de proposer un contenu à thématiser. La même remarque vaut, une troisième fois, pour $71 \mathrm{M}$.

D'autres reformulations ne sont que partielles, elles visent des buts différents: $74 \mathrm{E}$ «La taille de la botte blanche et noire.»

$75 \mathrm{M}$ «La taille...»

$76 \mathrm{E}$ «...d'une basket blanche.»

$[\ldots]$

$172 \mathrm{E} « 1,80$ fois $8 . »$

$173 \mathrm{M}$ «1,80 multiplié par... Donc vous, vous avez pris ici huit d'accord?»

[...]

$188 \mathrm{E}$ «On a calculé la taille euh... d'un homme...»

$189 \mathrm{M}$ «Oui, multiplié par...»

$190 \mathrm{E}$ «7,5, on a arrondi à $7 \ldots »$

$75 \mathrm{M}$ cherche à focaliser l'attention sur le génitif qui spécifie la taille, pour éviter une confusion lexicale potentiellement fâcheuse. La botte est en effet celle du géant, alors que ce qui est blanc et noir, ce sont les chaussures des hommes de l'image. 173M recourt également à une tournure plus appropriée: «fois» remplacé par «multiplié par». Mais cette intervention contribue surtout à rappeler aux élèves que le nombre 8 n'est que l'une des valeurs possibles du rapport botte / chaussure; le détachement d'une partie de l'énoncé initial contribue à lui conférer un statut de variable. $189 \mathrm{M}$ assure une fois encore cette précision du vocabulaire: «calculé» remplacé par «multiplié par». Mais de plus, la préposition «par» laissée en suspens provoque le rappel du coefficient de dilatation. Pour ces raisons, nous attribuerons à ces reformulations une force illocutoire directive: sans avoir l'air 
d'y toucher, le professeur amène ses interlocuteurs à compléter leurs tournures syntaxiques par l'ajout d'un complément du nom, d'un complément d'agent, ...

En même temps, puisqu'il ne fait que tendre la perche, puisqu'il se complait dans un rôle de provocateur qui relance sans cesse, l'enseignant reste neutre au plan cognitif: il ne fournit pas d'informations nouvelles, il n'introduit pas de thèmes inédits, ses interventions ne comportent quasiment pas d'assertifs. Il dévolue ainsi toute la responsabilité de la mésogenèse à la classe. Ses «on» sont purement rhétoriques (voir 67M ci-dessus), il ne s'agit pas d'un commissif de sa part, ce n'est pas lui qui s'engagera dans les calculs numériques. Son rôle est plus de reformuler les positions, en les paraphrasant au besoin, pour diffuser à l'ensemble de la communauté discursive les interventions de chacun.

Cela lui permet alors de stabiliser un certain nombre d'objets du milieu à travers ce qu'il note au tableau. Nous avons rendu compte dans le chapitre précédent de ces institutionnalisations partielles, locales. Si on s'y reporte, on verra que les trois volets du tableau assument des statuts fort différents. La partie centrale, la plus large, place sous les yeux de tous "Je cherche..." et "Je sais...». Une fois encore, de la part du maître, prise en charge énonciative par délégation, faux commissif: le locuteur, celui qui a tenu la craie pour écrire ces phrases, n'est pas l'énonciateur réel, celui qui sait et qui va chercher. La partie gauche quant à elle recense toutes les difficultés initiales (ce qu' "on» ne voit pas, ne sait pas, ...). Le volet droit servira à officialiser la méthode de résolution sur laquelle la classe s'entend finalement, sans aller néanmoins jusqu'à inscrire la solution numérique.

Une remarque encore sur ce dernier point. L'outil de résolution retenu, le produit en croix $(73 \mathrm{M}$, voir chapitre précédent), ne figure pas explicitement sur la fiche de préparation pédagogique. Il semble cependant devoir s'imposer, aux yeux de l'enseignant, parce que plusieurs groupes l'auraient utilisé précédemment. Un rapide sondage qu'il fait dans la classe lui montre cependant que moins de la moitié des élèves se rangent à sa position. Il la maintient néanmoins et organise le reste de la transaction à partir des quatre places que prévoit un tel tableau de proportionnalité. Exceptionnellement, ce ne sont pas les élèves qui provoquent des tensions entre dispositif et situation, mais le professeur lui-même, qui prend une initiative en se détachant de ce qu'il avait initialement prévu. Il active certains éléments de son "répertoire pédagogique», construit à travers sa formation et son expérience professionnelle. Son choix ne fait pas l'unanimité, puisque, malgré son institutionnalisation, seulement la moitié des apprenants utiliseront cette méthode dans leur écrit de résolution individuel final. De plus, quatre de ces onze élèves échoueront, alors que, chez ceux qu'il n'a pas réussi à convertir, dix sur onze parviendront à un résultat plausible. Il s'agit sans doute là d'un de ces cas de postulation mimétique que nous évoquions plus haut.

Le processus mésogénétique semble donc avoir été majoritairement pris en charge par les élèves. Mais, outre les remarques précédentes sur les interventions orales de l'enseignant, il faut souligner que ce dernier est encore bien présent à l'écrit. C'est en effet lui qui décide du moment où un thème est mûr pour être 
retranscrit, et c'est lui qui choisit l'emplacement où il le fera. Reprenons ces deux points. Chacun des énoncés correspond en fait à une sous-transaction; comme on l'a vu, l'ordre d'apparition des sous-thèmes importe peu au maître, seul compte le fait que la question a été abordée. On ne s'étonnera donc pas que ce qui figure finalement au tableau corresponde assez facilement à ce que prévoyait la fiche de préparation, à l'ordre près. Si ensuite on remonte des sous-transactions à la chronogenèse de l'ensemble de la séquence, on verra qu'il suffit de lire le tableau noir de la gauche vers la droite pour y apercevoir d'abord la liste de ce qui fait défaut, puis les valeurs numériques des mesures sur lesquelles la classe s'est accordée, et enfin la méthode de résolution finalement retenue.

Nous avons de la sorte analysé les interventions magistrales du point vue de la régulation des tensions qui apparaissent entre le dispositif conçu a priori et la situation réalisée ici et maintenant. Nous pouvons en rappeler le bilan en ce qui concerne les effets mésogénétiques obtenus.

S'agissant de la gestion des séances, nous retenons l'idée que les durées initialement prévues ont été respectées, ainsi que les alternances des modes de travail. L'enseignant obtient cela en assurant les opérations d'ouverture et de clôture des transactions. A l'inverse, au niveau sous-ordonné, c'est à la libre appréciation des élèves qu'il laisse le choix des sous-thématisations et, partant, de la prise en charge des interventions-initiatives. Là encore cependant, c'est lui qui intervient pour refermer une sous-transaction. Il se tient autant que faire se peut dans une stricte neutralité cognitive, par le truchement de reformulations littérales, ou paraphrastiques, ou partielles, selon ses intentions. Il dévolue de la sorte à ses élèves la responsabilité du processus de co-définition des objets sensibles du milieu pour apprendre.

Si l'on se focalise maintenant sur l'institutionnalisation, force est de reconnaître une fois encore que les prévisions ont été respectées: ce sont bien plusieurs procédures de résolution qui ont été officiellement explicitées et reconnues en fin de séquence. Intéressante entorse à la règle, un outil mathématique, le tableau de proportionnalité, a fait son apparition lors de cette synthèse finale, alors qu'il ne figurait pas en tant que tel sur la fiche de préparation. Nous faisons l'hypothèse de l'existence de répertoires pédagogiques disciplinaires construits sur la durée par les professeurs, dans lesquels ils vont puiser avec confiance au gré des circonstances. Notons pour finir que la structuration des acquis mésogénétiques est obtenue par les décisions de mise en page au tableau, elles aussi de la responsabilité de l'enseignant.

\section{Pour conclure: Chronogenèse, diégèse}

Nous voudrions pour commencer retenir les points suivants en guise de synthèse de notre étude. Comment l'enseignant s'y prend-il pour maitriser l'exploration d'un espace-problème par la classe afin que les élèves atteignent tout de même 
l'objectif fixé par le dispositif a priori? Par deux séries d'actions.

Les premières relèvent de la régulation des débats heuristiques. Le maître observé ici sélectionne le locuteur suivant, ce qui lui permet soit de renforcer une coalition d'élèves en accord sur un point donné, soit de faire apparaittre une coalition concurrente. Il maintient également l'orientation, écartant momentanément des propositions qui ne répondent pas à la consigne. Il ouvre et ferme les transactions, par changement de consigne justement. Il n'ouvre pas les sous-transactions, laissant la responsabilité des thématisations aux élèves, mais il les ferme en stabilisant les acquis.

La seconde série d'actions ressortit justement à ces institutionnalisations ponctuelles. L'enseignant commence par reformuler certains énoncés, à la fois pour recueillir l'assentiment de leur énonciateur et pour les diffuser à l'ensemble du groupe. Il va à l'occasion les faire relier à des acquis antérieurs. Il va finalement fixer les décisions collectives en les inscrivant au tableau. Dans le même mouvement, il va les structurer en les classant (les faits avec les faits, les questions avec les questions).

Dans ces conditions, les risques assumés par l'enseignant (voir ci-dessus) deviennent porteurs d'apprentissages, mettant les élèves en situation d'être actifs sans pour autant perdre le contrôle du déroulement de la séquence.

Nous voudrions pour finir soumettre au lecteur quelques remarques dépassant le seul corpus étudié ici. Le professeur observé n’a fixé aucune durée aux différentes transactions de la séquence au moment de sa préparation pédagogique. Il n'a fait que choisir le nombre et la durée des séances. Or, à chaque fois, il a fini exactement dans les temps, avec une alternance équilibrée des différentes phases. Son rôle relève donc en grande partie de la chronogenèse, on l'a rappelé ci-dessus, à travers les opérations d'ouverture / de fermeture des épisodes oraux. C'est-à-dire qu'il lui revient de «dilater / contracter» le temps en situation, de prendre en charge les répétitions, les analepses ou prolepses.

On aura reconnu là les trois catégories de phénomènes temporels identifiés par la narratologie (Genette, 1972):

- relations entre la durée des événements et la longueur du récit qui les relate;

- relations entre la fréquence d'un événement et le nombre de fois qu'il est mentionné par le récit;

- relations entre l'ordre des événements et l'ordre de leur présentation par le récit (voir Weisser, 2002, p. 150).

Spécifions:

- à quelques phrases jetées sur la fiche de préparation correspond un quart du temps de la séance, et inversement;

- quand un élève propose de développer sa démarche de résolution, l'enseignant le renvoie «à plus tard»;

- une sous-thématisation se superpose, s'enchevêtre à une autre, signe qu'entre la vingtaine d'élèves présents deux dialogues se poursuivent en parallèle. 
Mais ce qui distingue radicalement la narration de l'enseignement, c'est que, pour le roman, l'histoire (i.e. la succession des événements, réels ou fictifs) précède le récit, alors que dans la classe, le récit (i.e. le dispositif pédagogique a priori) cherche à anticiper ce que devra être l'histoire. Dans ces conditions, tous les efforts du maître tendront à faire coïncider la réalité qu'il est en train de faire advenir avec le récit qu'il en a écrit au préalable: il est d'abord le narrateur d'une histoire dont il sera ensuite l'un des protagonistes. On ne s'étonnera pas par conséquent d'observer certaines tensions entre dispositif et situation, dues à ce renversement de l'ordre du virtuel et du réel, à cette interversion entre diégèse et monde de l'action. Elles se trouvent de plus amplifiées par le fait que les autres parties prenantes des événements en jeu sont des personnes et non des personnages, parce que les élèves, "héros» du récit, ne sont pas soumis à l'autorité de l'auteur ou à l'omniscience du narrateur. C'est cette incertitude constitutive, cette tension entre le désiré et le réalisé, qui fait le sel du métier d'enseignant. C'est elle aussi qui dissuade le chercheur d'énoncer des lois nomothétiques à travers l'identification des causes de phénomènes reproductibles.

\section{Notes}

1 Cet article s'insère dans une recherche collective menée par le groupe ACODIS (Apprentissages en COntextes DIdactiqueS), composé de R. Adjiage, T. Beliaeva, R. Cabassut, N. Gavens, A. Jarlegan, J.-C. Rauscher, M.-J. Rémigy, M. Weisser, F. Wozniak.

2 Tous nos remerciements vont à B. Jacquel et à ses élèves, qui ont accepté de nous accueillir et se sont investis avec beaucoup de détermination dans l'étude du problème que nous leur avons soumis.

3 Photographie copiée de http://www.problempictures.co.uk/, avec l'aimable autorisation des auteurs.

4 Des analyses comparatives sont en cours, tant au niveau de la préparation préalable qu'à celui des situations vécues en classe et de leur efficacité.

5 Retranscription disponible auprès de Marc.Weisser@uha.fr.

$67 \mathrm{M}$ : intervention $\mathrm{n}^{\circ} 7$, locuteur: Maître.

\section{Bibliographie}

Adjiage, R. (2005). Diversité et invariants des problèmes mettant en jeu des rapports. Annales de Didactique et de Sciences Cognitives de l'IREM de Strasbourg, 10, 95-129.

Bernié, J.-P. (2002). L'approche des pratiques langagières scolaires à travers la notion de "communauté discursive»: Un apport à la didactique comparée? Revue française de pédagogie, 141, 77-88.

Bronckart, J.-P. (1996). Activité langagière, textes et discours. Pour un interactionnisme socio-discursif. Neuchâtel: Delachaux et Niestlé.

Brousseau, G. (1998). Théorie des situations didactiques. Grenoble: La Pensée Sauvage.

Charlier, P. \& Peeters, H. (1999). Contribution à une théorie du dispositif. Hermès, 25, $15-24$.

Cosnier, J. \& Kerbrat-Orecchioni, C. (Éd.). (1991). Décrire la conversation. Presses Universitaires de Lyon.

Forest, D. (2005). Une approche proxémique des interactions didactiques. Colloque "Didactiques: Quelle épistémologie?». IUFM d'Aquitaine et Université Victor Segalen Bordeaux II. 
Freinet, C. (1994). Les invariants pédagogiques. In C. Freinet, Euvres complètes (2 tomes) (pp. 383-418). Paris: Seuil.

Galison, P. (2002). Ainsi s'achèvent les expériences. Paris: La Découverte.

Genette, G. (1972). Figures III. Paris: Seuil.

Houssaye, J. (1988). Le triangle pédagogique. Berne: Peter Lang.

Johsua, S. (1996). Le concept de contrat didactique et l'approche vygotskienne. In C. Raisky \& M. Caillot (Éd.), Au-delà des didactiques, le didactique (pp. 145-158). Bruxelles: De Boeck.

Johsua, S. (2005). Conférence d'ouverture. Colloque "Didactiques: Quelle épistémologie?». IUFM d'Aquitaine et Université Victor Segalen Bordeaux II.

Leutenegger, F. \& Saada-Robert, M. (Éd.). (2002). Expliquer et comprendre en Sciences de l'Éducation. Bruxelles: De Boeck.

Lipman, M. (1995). A l'école de la pensée. Bruxelles: De Boeck.

Margolinas, C. (2005). Essai de généalogie en didactique des mathématiques. Revue suisse des sciences de l'éducation, 3, 343-360.

Matheron, Y. \& Salin, M. H. (2002). Les pratiques ostensives comme travail de construction d'une mémoire officielle de la classe dans l'action enseignante. Revue française de pédagogie, 141, 57-66.

Mercier, A., Schubauer-Leoni, M. L. \& Sensevy, G. (2002). Vers une didactique comparée. Revue française de pédagogie, 141, 5-16.

Meunier, J. P. (1999). Dispositif et théories de la communication. Hermès, 25, 83-92.

Moeschler, J. \& Reboul, A. (1994). Dictionnaire encyclopédique de pragmatique. Paris: Seuil.

Nonnon, E. (1996). Activités argumentatives et élaboration de connaissances nouvelles: Le dialogue comme espace d'exploration. Langue Française, 112, 67-87.

Orange, C. (2004), Argumentation et activités de recherche. In J. Douaire (Éd.), Argumentation et disciplines scolaires (pp.13-18). Paris: INRP.

Rauscher, J. C., Adjiage, R. \& Beliaeva, T. (2009). Modélisation et écrits réflexifs: Des outils pour apprendre? Actes du 37e Colloque de la COPIRELEM, 9-11 juin 2010, Auch.

Sartre, J. P. (1943). L'Être et le Néant. Paris: Gallimard.

Schubauer-Leoni, M. L. (1994). Quatre élèves et un problème de distances: Approche didactique de l'analyse des interactions. In A. Trognon, U. Dausendschön-Gay, U. Krafft \& C. Riboni (Éd.), La construction interactive du quotidien (pp. 77-102). Presses Universitaires de Nancy.

Schubauer-Leoni, M. L. (1996). Étude du contrat didactique pour des élèves en difficulté en mathématiques. In C. Raisky \& M. Caillot (Éd.), Au-delà des didactiques, le didactique (pp. 159-192). Bruxelles: De Boeck.

Schubauer-Leoni, M. L. (2003). La fonction des dimensions langagières dans un ensemble de travaux sur le contrat didactique. Actes du colloque "Construction des connaissances et langage dans les disciplines d'enseignement». Université de Bordeaux, IUFM d'Aquitaine.

Schubauer-Leoni, M. L. \& Chiesa Millar, V. (2002). Une «tâche de français sur un thème de géographie»: Actions didactiques de l'enseignante dans le vif de l'activité en classe et dans son discours a priori. Revue française de pédagogie, 141, 123-134.

Sensevy, G. (2001). Modèles pour l'action du professeur: Nécessités, difficultés. In A. Mercier, G. Lemoyne \& A. Rouchier (Éd.), Le génie didactique. Usages et mésusages des théories de l'enseignement (pp. 209-232). Bruxelles: De Boeck.

Sensevy, G., Mercier, A. \& Schubauer-Leoni, M. L. (2000). Vers un modèle de l'action didactique du professeur: À propos de la Course à 20. Recherches en didactique des mathématiques, 20, (3), 263-304.

Sensevy, G. \& Quilio, S. (2002). Le discours du professeur. Vers une pragmatique didactique. Revue française de pédagogie, 141, 47-56.

Swales, J. M. (1990). Genre analysis. English in academic and research settings. Cambridge: University Press. 
Trognon, A. (1994). Théories et modèles de la construction interactive du quotidien. In A. Trognon, U. Dausendschön-Gay, U. Krafft \& C. Riboni (Éd.), La construction interactive du quotidien (pp. 7-52). Presses Universitaires de Nancy.

Trognon, A. (1999). Éléments d'analyse interlocutoire. In M. Gilly, J.-P. Roux \& A. Trognon (Éd.), Apprendre dans l'interaction (pp. 69-94). Presses Universitaires de Nancy et Publications de l'Université de Provence.

Trognon, A., Dausendschön-Gay, U., Krafft, U. \& Riboni, C. (1994). La construction interactive du quotidien. Presses Universitaires de Nancy.

Vion, R. (1999). Linguistique et communication verbale. In M. Gilly, J.-P. Roux \& A. Trognon (Éd.), Apprendre dans l'interaction (pp. 41-69). Presses Universitaires de Nancy et Publications de l'Université de Provence.

Vygotsky, L. S. (1934/1985). Pensée et langage. Paris: Messidor.

Weisser, M. (2002). La lecture à l'école et au collège: Entre psittacisme et délire. Paris: L'Harmattan.

Weisser, M. (2003). La gestion didactique des situations d'argumentation orale. Les Sciences de l'éducation - Pour l'Ère Nouvelle, 36, (3), 49-76.

Weisser, M. (2004). Langage et apprentissages dans l'Émile de Jean-Jacques Rousseau. Penser l'éducation, 15, 103-121.

Weisser, M. (2006). Comprendre et interpréter un texte narratif de fiction: Un exemple d'argumentation heuristique au Cycle III. Carrefours de l'éducation, 21, 31-52.

Weisser, M. (2007). Méthodes d'analyse des interactions verbales au service d'une didactique comparée. Revue française de pédagogie, 158, 103-115.

Weisser, M. (2008). Au confluent de la didactique et de la pédagogie: Le processus Apprendre. Penser l'éducation, 23, 101-116.

Weisser, M. (2009). Espaces didactiques: Conditions micro sociales de l'apprentissage. Éducation et Didactique, 3, (2), 101-112.

Weisser, M. (2010). Situation. Le Télémaque, 37, 15-30.

Mots clés: Didactique des mathématiques, didactique comparée, dispositifs d'enseignement, interactions orales, analyse de discours

\section{Unterrichtssteuerung und mesogenetische Effekte auf den Wissenserwerb: Spannungsfeld zwischen Unterrichtsplanung und -situation}

\section{Zusammenfassung}

In diesem Beitrag geht es um die Frage, wie eine Lehrperson gleichzeitig den Austausch zwischen Schülern/innen regulieren und seine Unterrichtsziele erreichen kann. Wir vergleichen den Verlauf einer Unterrichtssequenz mit der darauf bezogenen, a priori vorgenommenen Planung. Das gesammelte Material wurde mit den Methoden der pragmatischen Linguistik analysiert. Dabei wurden strategische Passagen identifiziert, in denen die Lehrperson reflektierend in das Geschehen der Klasse eingreift und lokale, partielle Zwischenergebnisse sichert. In den Schlußfolgerungen werden einige Überlegungen vorgestellt, die sich auf die Spannung zwischen der Unterrichts $\neg$ planung und dem aktuellen Erleben in der Klasse beziehen. 
Schlagworte: Didaktik der Mathematik, vergleichende Didaktik, Unterrichtsplanung, verbale Interaktionen; Diskursanalyse

\section{Regolazioni dell'insegnamenti ed effetti mesogenetici: tensioni tra dispositivo e situazione.}

\section{Riassunto}

In che modo l'insegnante regola gli scambi tra allievi per raggiungere i suoi obiettivi? Questa è la domanda che ci proponiamo di approfondire in questo testo. Per farlo, confrontiamo lo svolgimento di una sequenza d'insegnamento con il dispositivo concepito a priori dal docente. Gli strumenti utilizzati per analizzare il corpus raccolto provengono dalla pragmatica linguistica. Vengono identificati i luoghi strategici nei quali l'insegnante interviene per orientare la riflessione della sua classe, e vengono precisati i ruoli definiti dalle sue istituzionalizzazioni locali e parziali. Le conclusioni presentano delle riflessioni sulle tensioni tra dispositivo pedagogico e situazione vissuta in aula.

Parole chiave: Didattica della matematica, didattica comparata, dispositivi d'insegnamento, interazioni verbali, analisi del discorso

\section{Classroom lecturing regulations and mesogenetic effects: Tensions between teaching devices and situation}

\section{Summary}

How does a teacher manage to regulate the exchanges between pupils in order to achieve his goals? That is the question we propose to examine within this contribution. We compare the course of a teaching sequence with the device previously designed by the professor. The tools implemented to analyze the collected corpus are borrowed from pragmatic linguistics. The strategic places where the teacher intervenes to direct the reflection of his class are identified. The role played by its local and partial institutionalizations is specified. The conclusion presents some suggestions about the tensions between the preexistent teaching device and the situation lived into the classroom.

Keywords: Didactics of mathematics, comparative didactics, teaching devices, verbal interactions, discourse analysis 
